\begin{tabular}{|r|l|}
\hline \multicolumn{2}{|c|}{ Statistica Sinica Preprint No: SS-2021-0018 } \\
\hline Title & $\begin{array}{l}\text { An Online Projection Estimator for Nonparametric } \\
\text { Regression in Reproducing Kernel Hilbert Spaces }\end{array}$ \\
\hline Uanuscript ID & SS-2021-0018 \\
\hline URL & http://www.stat.sinica.edu.tw/statistica/ \\
\hline DOI & $10.5705 /$ ss.202021.0018 \\
\hline Complete List of Authors & $\begin{array}{l}\text { Tianyu Zhang and } \\
\text { Noah Simon }\end{array}$ \\
\hline Corresponding Author & Tianyu Zhang \\
\hline E-mail & zty@uw.edu \\
\hline Notice: Accepted version subject to English editing.
\end{tabular}


Statistica Sinica

\title{
An Online Projection Estimator for Nonparametric Regression in Reproducing Kernel Hilbert Spaces
}

\author{
Tianyu Zhang and Noah Simon \\ University of Washington
}

\begin{abstract}
The goal of nonparametric regression is to recover an underlying regression function from noisy observations, under the assumption that the regression function belongs to a pre-specified infinite dimensional function space. In the online setting, when the observations come in a stream, it is generally computationally infeasible to refit the whole model repeatedly. There are as of yet no methods that are both computationally efficient and statistically rate-optimal. In this paper, we propose an estimator for online nonparametric regression. Notably, our estimator is an empirical risk minimizer (ERM) in a deterministic linear space, which is quite different from existing methods using random features and functional stochastic gradient. Our theoretical analysis shows that this estimator obtains rate-optimal generalization error when the regression function is known to live in a reproducing kernel Hilbert space. We also show, theoretically and empirically, that the computational expense of our estimator is much lower than other rate-optimal estimators proposed for this online setting.
\end{abstract}

Key words and phrases: nonparametric regression, online learning, reproducing kernel Hilbert space, Mercer expansion 


\section{Introduction}

It is often of interest to estimate an underlying regression function, linking features to an outcome, from noisy observations. In the case that the structure of this function is not known (e.g. when we do not want to assume a simple linear form), some form of nonparametric regression is employed. More formally, suppose we observe $\left(X_{i}, Y_{i}\right) \stackrel{i . i . d .}{\sim} \rho(X, Y), i=1,2, \ldots, n$ generated from the following statistical model:

$$
Y_{i}=f_{\rho}\left(X_{i}\right)+\epsilon_{i}
$$

where, for each $i, X_{i} \stackrel{i . i . d .}{\sim} \rho_{X}$ (which take value in $\mathbb{R}^{d}$ ) are our features, $Y_{i} \in \mathbb{R}$ is our outcome, $\epsilon_{i}$ are iid mean 0 noise variables. One can think of $f_{\rho}$ as implicitly defined by the joint distribution $\rho(X, Y)$. It is often of interest to estimate $f_{\rho}$, the regression function (e.g. in predictive modeling, or inferential applications). Under mild conditions, the regression function $f_{\rho}$ can also be characterized as the minimizer of

$$
\min _{f \in \mathcal{F}} \mathbb{E}(Y-f(X))^{2}
$$

when $\mathcal{F}=L_{\rho_{X}}^{2}$, which is the best measurable function for predicting $Y$ given $X$ under least squares loss. 
1.1 Nonparametric Regression in RKHS3

\subsection{Nonparametric Regression in RKHS}

In nonparametric regression we often assume that $f_{\rho}$ belongs to a specified infinite dimensional function space $\mathcal{F}$. This is known as the Hypothesis Space. Some commonly used $\mathcal{F}$ in statistics and computer science communities are the Holder ball, Sobolev spaces (Wahba, 1990), general reproducing kernel Hilbert spaces (RKHS) (Christmann and Steinwart, 2008), and Besov spaces (Härdle et al., 2012). In this paper, we focus on estimation when $\mathcal{F}$ is a RKHS. Briefly, a RKHS over $\mathcal{X}$ is a Hilbert space $\left(\mathcal{F},\langle\cdot, \cdot\rangle_{\mathcal{F}}\right)$ with the reproducing property: for any $f \in \mathcal{F}, x \in \mathcal{X}$,

$$
f(x)=\left\langle f, K_{x}\right\rangle_{\mathcal{F}}
$$

where $K_{x}$ is the so-called kernel function associated with $\mathcal{F}$ evaluated at $x$. This is discussed in more detail in Section 2 ,

In the classical non-streaming setting of nonparametric regression, estimation in a RKHS $\mathcal{F}$ is a well-studied problem. In this case, the kernel ridge regression (KRR) estimator is the gold standard, e.g. (Wainwright, 2019). It is defined by

$$
\hat{f}_{n}^{K R R}:=\underset{f \in \mathcal{F}}{\operatorname{argmin}} \frac{1}{n} \sum_{i=1}^{n}\left(Y_{i}-f\left(X_{i}\right)\right)^{2}+\lambda_{n}^{K R R}\|f\|_{\mathcal{F}}^{2}
$$

where $\lambda_{n}^{K R R}$ is a hyper-parameter that balances the mean-square error and the complexity of estimate. Thanks to the reproducing property 1.3, 
1.2 Parametric and Nonparametric Online Learning4

$\hat{f}_{n}^{K R R}$ can be written as a finite linear combination of the kernel function evaluated at $\left(X_{i}\right)_{i=1}^{n}$ (Schölkopf et al. 2001$)$.

In general (1.4) requires solving an $n \times n$ linear system, and thus, will have a computational expense on the order of $n^{3}$. In the online setting, this is exacerbated by the need to refit for each new observation resulting in $n^{4}$ computation required to fit a sequence of $n$ estimators. While this penalized estimator has good statistical properties (rate optimal convergence and strong empirical performance), the high computational expense restricts its application in the online setting. Substantial effort has been spent on reducing the computational expense of KRR using, for example, "scalable kernel machines" based on random Fourier feature (RFF) (Liu et al., 2020) or Nyström projection (Gittens and Mahoney, 2016). This is further discussed in Section 2.1.

\subsection{Parametric and Nonparametric Online Learning}

Online learning has been thoroughly studied in the parametric setting: there we assume $f_{\rho}$ takes a parametric form indexed by a finite dimensional parameter $\beta \in \mathbb{R}^{p}$ (e.g. $f_{\rho}(X)=\beta^{\top} X$ for a linear model).

In this parametric online setting, it is useful to frame the regression 


\subsection{Parametric and Nonparametric Online Learning5}

function as a population minimizer

$$
\min _{\beta \in \mathbb{R}^{p}} \mathbb{E}\left[\left(Y-f_{\beta}(X)\right)^{2}\right]
$$

From here, it is popular to directly apply stochastic gradient descent (SGD) to (1.5), by using each sample in our "stream" to calculate one unbiased estimate of the gradient. Updating such an estimator with a new observation has constant computational expense, $O(p)$. Additionally, these estimators achieve the optimal parametric convergence rate $O(1 / n)$ under mild conditions (Kushner and Yin, 2003; Bach and Moulines, 2013; Frostig et al., 2015; Babichev and Bach, 2018).

However, comparatively less attention has been given to online nonparametric regression. A few rate-optimal functional stochastic gradient descent algorithms have been proposed in the last decade (Tarres and Yao, 2014; Dieuleveut and Bach, 2016), where the hypothesis function space $\mathcal{F}$ is assumed to be a RKHS. The RKHS structure makes it possible to take the gradient of the evaluation functional $L_{x}(f):=f(x)$. Although such estimators have been shown to be statistically rate-optimal, updating them with a new observation $\left(X_{n+1}, Y_{n+1}\right)$ usually involves evaluating $n$ kernel functions at $X_{n+1}$, with computational expense of order $O(n)$. This is in contrast with the constant update cost of $O(p)$ in parametric SGD. Thus, the computational cost of nonparametric SGD will accumulate at order $O\left(n^{2}\right)$, which 
1.2 Parametric and Nonparametric Online Learning6

is not ideal for methods that are nominally designed to deal with large datasets. Although there has been some effort devoted to transfer RFF- or Nystrom- based methods to the online setting (See Section 2.1), the theoretical guarantees are usually not close to optimal with strong restrictions on the noise variables.

Our contribution In this paper, we propose a method for constructing online estimators in a RKHS by considering the Mercer expansion (eigendecomposition) of a kernel function. Existing methods usually takes an iterative form, which can be interpreted as projecting a random function onto a random space with growing dimension (Koppel et al., 2019, Equation (15)). However, our estimator is the first one that can be treated as an empirical risk minimizer (ERM, or M-estimator of negative loss) in a deterministic linear space with growing dimension.

Analysis of both the statistical and the computational properties of the estimator is performed to show that: i) it has asymptotically optimal (up to a logarithm term) generalization error; ii) it has significantly lower computational expense than other proposed rate-optimal nonparametric SGD estimators; iii) it is robust against heavy-tailed noise. Interestingly, it only requires the $(1+\Delta)$ moment of the noise to be finite for any $\Delta>0$ to achieve consistency. 
It is worth noting that in the theoretical analysis of our estimator, we do not require the covariate $X$ to be equally-spaced or uniformly distributed as in standard references (Tsybakov, 2008) (though such assumptions could significantly simplify the proof). We additionally do not require it to be known for rate optimal convergence. We show that our estimator will obtain rate optimal convergence if $\rho_{X}$ is absolutely continuous with respect to the measure that is used to conduct the eigendecomposition of the kernel function (usually the latter is taken as uniform measure or a Gaussian distribution).

Notation: we use $a_{n}=\Theta\left(b_{n}\right)$ to indicate that the two sequences increase/decrease at the same rate as $n \rightarrow \infty$. Formally,

$$
0<\liminf _{n \rightarrow \infty}\left|\frac{a_{n}}{b_{n}}\right| \leq \limsup _{n \rightarrow \infty}\left|\frac{a_{n}}{b_{n}}\right|<\infty
$$

For $a \in \mathbb{R},\lfloor a\rfloor$ is the largest integer that is smaller than or equal to $a$. The $\|\cdot\|_{2}$-norm of a function is its $L_{\rho_{X}}^{2}$-norm, i.e $\|f\|_{2}^{2}=\int_{\mathcal{X}} f^{2}(z) d \rho_{X}(z)$. In this paper, by saying two functions $f, g$ are orthogonal with respect to measure $P$, we mean $\int f(x) g(x) d P(x)=0$.

\section{Preliminaries on RKHS}

In this section, we are going to provide background information on RKHS and existing methods before introducing our estimation procedure. 
First we formally introduce the concept of Mercer kernel and its corresponding RKHS. A symmetric bivariate function $K: \mathcal{X} \times \mathcal{X} \rightarrow \mathbb{R}$ is positive semi-definite (PSD) if: for any $n \geq 1$ and $\left(x_{i}\right)_{i=1}^{n} \subset \mathcal{X}$, the $n \times n$ kernel matrix $\mathbb{K}$ whose elements are $\mathbb{K}_{i j}:=K\left(x_{i}, x_{j}\right)$ is always a PSD matrix. A continuous, bounded, PSD kernel function $K$ is called a Mercer kernel. We have the following duality between a Mercer kernel and a Hilbert space:

Proposition 1. For any Mercer Kernel $K: \mathcal{X} \times \mathcal{X} \rightarrow \mathbb{R}$, let $K_{x}$ denote the function $K_{x}(\cdot):=K(x, \cdot)$. There exists an unique Hilbert Space $\left(\mathcal{H},\langle\cdot, \cdot\rangle_{\mathcal{H}}\right)$ of functions on $\mathcal{X}$ satisfying the following conditions.

1. For all $x \in \mathcal{X}, K_{x} \in \mathcal{H}$.

2. The linear span of $\left\{K_{x} \mid x \in \mathcal{X}\right\}$ is dense (w.r.t $\left.\|\cdot\|_{\mathcal{H}}\right)$ in $\mathcal{H}$

3. (reproducing property) For all $f \in \mathcal{H}, x \in \mathcal{X}$,

$$
f(x)=\left\langle f, K_{x}\right\rangle_{\mathcal{H}}
$$

We call this Hilbert space the Reproducing kernel Hilbert space (RKHS) associated with kernel $K$, or the native space of $K$. For a more comprehensive discussion of RKHS, see Cucker and Smale (2002); Wainwright (2019); Fasshauer and McCourt (2015).

There is an equivalent definition of RKHS that we will engage with in this manuscript. Given any Mercer kernel $K$ and any Borel measure 
$\nu$, there exists a set of $L_{\nu}^{2}$-orthonormal basis $\left(\phi_{j}\right)_{j=1}^{\infty}$ of $\overline{\mathcal{H}}$ (closure of $\mathcal{H}$ with respect to $\|\cdot\|_{L_{\nu}^{2}}$. Additionally, each of the functions has a paired positive real number $\mu_{j}$, sorted s.t. $\mu_{j} \geq \mu_{j+1}>0$. We call the functions $\phi_{j}$ 's eigenfunctions and $\mu_{j}$ 's their corresponding eigenvalues. We state the following equivalent definition of the native space of $K$.

Proposition 2. Define a Hilbert space

$$
\mathcal{H}=\left\{f \in L_{\nu}^{2} \mid f=\sum_{k=1}^{\infty} \theta_{j} \phi_{j} \text { with } \sum_{j=1}^{\infty}\left(\frac{\theta_{j}}{\sqrt{\mu_{j}}}\right)^{2}<\infty\right\}
$$

equipped with inner product:

$$
\langle f, g\rangle_{\mathcal{H}}=\sum_{j=1}^{\infty} \frac{a_{j} b_{j}}{\mu_{j}}
$$

for $f=\sum_{j=1}^{\infty} a_{j} \phi_{j}$ and $g=\sum_{j=1}^{\infty} b_{j} \phi_{j}$.

Then $\left(\mathcal{H},\langle\cdot, \cdot\rangle_{\mathcal{H}}\right)$ is the reproducing Hilbert space of kernel $K$.

For discussion of this definition and its relation to Proposition 1, see Cucker and Smale (2002). For many kernels, the analytical form of the $\left(\mu_{j}, \phi_{j}\right)$ 's are available for some specific choice of measure $\nu$. This can be quite useful for our method: We require the eigen-system of the kernel with respect to some (relatively arbitrary) measure. This measure does not need to be the measure $\rho_{X}$, it merely needs to be absolutely continuous with respect to $\rho_{X}$. In this manuscript we will assume such a convenient measure, 
denoted, $\bar{\rho}_{X}$ exists (for which the kernel has an accessible eigen-system and $\left.\bar{\rho}_{X} \ll \rho_{X}\right)$. We will call it a working measure, and use the notation $\left(\lambda_{j}, \psi_{j}\right)$ instead of the generic $\left(\mu_{j}, \phi_{j}\right)$ to denote such an eigen-system with respect to $L_{\bar{\rho}_{X}}^{2}$. As an example, the kernel $K(x, z)=\min \{x, z\}$ is the reproducing kernel of Sobolev space

$$
W_{1}^{0}([0,1])=\left\{f:[0,1] \rightarrow \mathbb{R} \mid f(0)=0 \text { and } \int_{0}^{1}\left(f^{\prime}(x)\right)^{2} d x<\infty\right\}
$$

and its eigenfunctions and eigenvalues are (w.r.t. $\left.\bar{\rho}_{X}=\operatorname{Unif}([0,1])\right)$ :

$$
\psi_{j}(x)=\sqrt{2} \sin \left(\frac{(2 j-1) \pi x}{2}\right) \quad \lambda_{j}=\frac{4}{(2 j-1)^{2} \pi^{2}}
$$

It is also possible to write the kernel as a Mercer expansion w.r.t $\left(\psi_{j}, \lambda_{j}\right)$ :

$$
K(x, z)=\sum_{j=1}^{\infty} \lambda_{j} \psi_{j}(x) \psi_{j}(z)
$$

The functions $\left\{\sqrt{\lambda_{j}} \psi_{j}(x), j=1,2, \ldots\right\}$ are also called the feature maps of the kernel $K$. Also note that by definition $\psi_{j}{ }^{\prime}$ 's are orthogonal w.r.t. $\langle\cdot, \cdot\rangle_{\mathcal{H}}$. There is a collection of 20 commonly used kernels' Mercer expansion in (Fasshauer and McCourt, 2015, Appendix A).

If a function $f=\sum_{j=1}^{\infty} \theta_{j} \psi_{j}$ has a finite $\|\cdot\|_{\mathcal{H}}$ RKHS-norm. Its general Fourier coefficients $\left(\theta_{j}\right)_{j \in \mathbb{N}}$ need to be at least $o\left(\lambda_{j} j^{-1 / 2}\right)$ so that the norm series $\sum_{j=1}^{\infty}\left(\theta_{j} / \sqrt{\lambda_{j}}\right)^{2}$ converges. This suggests, for sufficiently large $N$, the truncation $f_{N}=\sum_{j=1}^{N} \theta_{j} \psi_{j}$ should be a good approximation to $f$. This 
2.1 Existing Online Nonparametric Methods11

basic idea motivates our work - by analyzing the spectrum of the kernel we can identify what $N$ should be.

\subsection{Existing Online Nonparametric Methods}

In a RKHS, it is possible to take the functional gradient of the evaluation operator $L_{x}$ for any $x \in \mathcal{X}$. This allows methods using functional SGD to solve the regression problem 1.2 . Usually functional $S G D$ estimators after $n$ steps, $\hat{f}_{n}^{S G D}$ of $f_{\rho}$ take the form of a weighted sum of $n$ kernel functions $K_{X_{i}}, i=1,2, \ldots, n$, (Tarres and Yao, 2014; Dieuleveut and Bach, 2016):

$$
\hat{f}_{n}^{S G D}=\sum_{i=1}^{n} a_{i} K_{X_{i}}
$$

To update $\hat{f}_{n}^{S G D}$ with $\left(X_{n+1}, Y_{n+1}\right)$, it is necessary to evaluate all $n$ kernel basis functions $\left\{K_{X_{i}}, i=1,2, \ldots n\right\}$ at $X_{n+1}$. Thus, the computational expense of the update is $O(n)$. There has been some work to improve this computational expense: in Si et al. (2018); Lu et al. (2016); Koppel et al. (2019), the authors choose a subset of features $\left(K_{X_{i}}\right)_{i=1}^{n}$ whose cardinality is smaller than $n$; in Dai et al. (2014); Lu et al. (2016), kernel-agnostic random Fourier features are used: Typically $O(\sqrt{n})$ basis functions are required in this setting, cf. Rudi and Rosasco (2017). Although computationally more efficient than vanilla functional SGD 2.13 , the theoretical aspects of these scalable methods are not fully satisfying: 1) noise variables are required 
2.1 Existing Online Nonparametric Methods12

to have extreme light tails to provably guarantee convergence; 2) Verified convergence rates are not minimax-optimal; and 3) The target parameter is generally not even $f_{\rho}$ but, instead, a penalized population risk-minimizer.

Compared with the linear space spanned by random features or kernel functions (engaged with in previous work), the space spanned by eigenfunctions has minimal approximation error in the sense of minimizing Kolmogorov N-width (Santin and Schaback, 2016, Section 3). This inspired us to use them as basis functions to construct our estimator. Briefly, this means projecting onto the $\mathrm{N}$-dimension linear space spanned by the eigenfunctions has the minimal residual, among all the N-dimension linear subspaces of $L_{\bar{\rho}_{X}}^{2}$. More technically:

$\sup _{\|f\|_{\mathcal{H}}=1}\left\|f-\Pi_{L_{\bar{\rho}_{X}}^{2}, \mathcal{F}_{N}} f\right\|_{L_{\bar{\rho}_{X}}^{2}}=\inf _{V_{N} \subset L_{\bar{\rho}_{X}}^{2}} \sup _{\|f\|_{\mathcal{H}}=1}\left\|f-\Pi_{L_{\bar{\rho}_{X}}^{2}, V_{N}} f\right\|_{L_{\bar{\rho}_{X}}^{2}}=\sqrt{\lambda_{N+1}}$

where $\mathcal{F}_{N}$ is the linear space spanned by the first $N$ eigenfunctions $\left(\psi_{j}\right)_{j=1}^{N}$, $\Pi_{A, B}$ is the projection operator onto space $B$ using the inner product of $A$ and $V_{N}$ is a generic $N$-dimension linear space in $L_{\bar{\rho}_{X}}^{2}$. This is important for statistical estimation as there is a bias/variance tradeoff at play in this estimation problem (more basis functions decreases bias but increases variance). By using a basis that can more compactly represent our function, we can find a more favorable tradeoff and asymptotically decrease our 
estimation error.

Our research aims to propose a method with favorable statistical guarantees (minimax rate-optimality) and a lower computational expense. The basis functions used should be kernel-sensitive and the convergence rate should be sensitive to the decay rate of eigenvalues $\lambda_{j}$. Also, we give provable theoretical guarantees in a heavy tail noise setting.

\section{A Computationally Efficient Online Estimator}

In this section we will present the proposed online regression estimator. We first discuss the well-known projection estimator in the batch learning setting, then shift to the online setting where we naively refit the model with each observation; and finally give our proposed modification to make this process computationally efficient. In what follows we will use $N$ to denote the number of basis functions used to construct each projection estimator, though it should more formally be written as $N(n)$, as it is a non-decreasing function of $n$.

\subsection{Projection Estimator in Batch Learning}

Suppose we have $n$ samples $\left(X_{i}, Y_{i}\right)_{i=1}^{n}$, and let $\mathcal{F}_{N}=\operatorname{span}\left(\psi_{1}, \ldots, \psi_{N}\right)$ be the $N$-dimension linear space spanned by the $N$ eigenfunctions with largest 
3.1 Projection Estimator in Batch Learning14

eigenvalues. The function $\hat{f}_{n, N}$ that minimizes empirical mean square error over $\mathcal{F}_{N}$ is a very attractive candidate for estimating $f_{\rho} \in \mathcal{H}$, that we aim to leverage for the online setting.

Formally, define $\boldsymbol{\theta}=\left(\theta_{1}, \ldots, \theta_{N}\right)^{\top}$ and $\boldsymbol{\psi}^{N}\left(X_{i}\right)=\left(\psi_{1}\left(X_{i}\right), \ldots, \psi_{N}\left(X_{i}\right)\right)^{\top}$. Consider the least squares problem (in Euclidean space):

$$
\min _{\boldsymbol{\theta} \in \mathbb{R}^{N}} \sum_{i=1}^{n}\left(Y_{i}-\boldsymbol{\theta}^{\top} \boldsymbol{\psi}^{N}\left(X_{i}\right)\right)^{2}
$$

The solution can be written in matrix form as

$$
\hat{\boldsymbol{\theta}}:=\left(\hat{\theta}_{1}, \ldots, \hat{\theta}_{N}\right)^{\top}=\left(\Psi_{n}^{\top} \Psi_{n}\right)^{-1} \Psi_{n}^{\top} \boldsymbol{Y}_{n}
$$

if $\Psi_{n}^{\top} \Psi_{n}$ is invertible. Here $\boldsymbol{Y}_{n}=\left(Y_{1}, \ldots, Y_{n}\right)^{\top}$ is the observed response, and $\Psi_{n}$ is the design matrix whose elements are $\Psi_{i j}=\psi_{j}\left(x_{i}\right)$. Then the estimator

$$
\hat{f}_{n, N}=\sum_{j=1}^{N} \hat{\theta}_{j} \psi_{j}
$$

is the empirical risk minimizer $(\mathrm{ERM})$ in $\mathcal{F}_{N}$. Estimators that take this form are called nonparametric projection estimators (of $f_{\rho}$, with level $N$ ) (Tsybakov, 2008).

The optimal number of basis functions to use depends on both the sample size $n$ and how fast the eigenvalues $\lambda_{j}$ in $(2.12)$ decay. As we will state formally in Theorem 3 , the optimal choice is $N=\Theta\left(n^{\frac{d}{2 \alpha+d}}\right)$ when $\lambda_{j}=\Theta\left(j^{-2 \alpha / d}\right)$, with $\alpha>\frac{d}{2}$. Note that the condition $\alpha>\frac{d}{2}$ ensures 
the considered RKHS can be embedded into the space of continuous functions (as a result of Sobolev inequality, cf. Theorem 12.55 (Leoni, 2017)). With this choice for $N$, convergence of $\hat{f}_{n, N}$ achieves the minimax rate over functions with bounded RKHS norm. Similar results for projection estimators have been shown when $\left(\psi_{j}\right)_{j=1}^{\infty}$ is the trigonometric basis, and $x_{i}$ are deterministic, and evenly spaced (Tsybakov, 2008) or $\rho_{X}$ is the uniform distribution (Belloni et al., 2014). Our analysis shows that the optimality of the projection estimator actually holds for general $\psi_{j}$ and does not require them to be orthonormal with respect to the empirical measure or $\rho_{X}$.

\subsection{Naive Online Projection Estimator}

The most direct way of extending the projection estimator (3.17) to the online setting is simply refitting the whole model whenever a new pair of data $\left(X_{i}, Y_{i}\right)$ comes in. In Algorithm 1, we provide this naive updating rule for our reader to better understand the proposed method. Our modified proposal in Section 3.4 greatly improves upon this in terms of computational cost but giving the same estimates $\hat{f}_{n, N}$.

In this algorithm, $Y_{n}=\left(Y_{1}, \ldots, Y_{n}\right)^{\top}$ is the vector of outcomes. $\Psi_{n}$ is the $n \times N$ design matrix at step $n$, and $\Phi_{n}$ denotes the $N \times N$ matrix $\left(\Psi_{n}^{\top} \Psi_{n}\right)^{-1}$ (inversion of Gram matrix). 


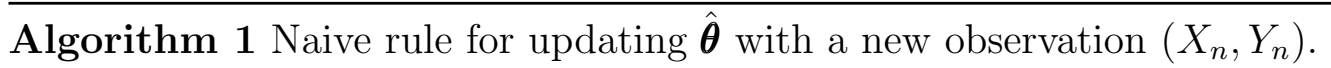
Input: $\left(X_{i}\right)_{i=1}^{n}, \boldsymbol{Y}_{n}, \Phi_{n-1}, \Psi_{n-1}, \alpha, N$

function UpdateCurrent $\left(X_{n}, N, \Phi_{n}, \Psi_{n}\right)$

$$
\begin{aligned}
& \boldsymbol{\psi}_{n} \leftarrow\left[\psi_{1}\left(X_{n}\right), \psi_{2}\left(X_{n}\right), \ldots, \psi_{N}\left(X_{n}\right)\right]^{\top} \\
& \Psi_{n} \leftarrow\left[\begin{array}{l}
\Psi_{n} \\
\boldsymbol{\psi}_{n}^{\top}
\end{array}\right] \quad \Phi_{n} \leftarrow\left(\Psi_{n}^{\top} \Psi_{n}\right)^{-1} \\
& \text { return }\left(\Phi_{n}, \Psi_{n}\right)
\end{aligned}
$$

function AddBasis $\left(\left(X_{i}\right)_{i=1}^{n}, N, \Phi_{n}, \Psi_{n}\right)$

$$
\begin{aligned}
& \boldsymbol{\psi}^{N+1} \leftarrow\left[\psi_{N+1}\left(X_{1}\right), \ldots, \psi_{N+1}\left(X_{n}\right)\right]^{\top} \\
& \Psi_{n} \leftarrow\left[\begin{array}{ll}
\Psi_{n} & \left.\psi^{N+1}\right]
\end{array}\right] \quad \Phi_{n} \leftarrow\left(\Psi_{n}^{\top} \Psi_{n}\right)^{-1} \\
& \text { return }\left(\Phi_{n}, \Psi_{n}\right) \\
& \text { if } n=\operatorname{Floor}\left((N+1)^{2 \alpha+1}\right) \text { then } \\
& \qquad\left(\Phi_{n}, \Psi_{n}\right) \leftarrow \operatorname{UpdateCurrent}\left(X_{n}, N, \Phi_{n-1}, \Psi_{n-1}\right) \\
& \quad\left(\Phi_{n}, \Psi_{n}\right) \leftarrow \operatorname{AddBasis}\left(\left(X_{i}\right)_{i=1}^{n}, N, \Phi_{n}, \Psi_{n}\right) \\
& \quad N \leftarrow N+1
\end{aligned}
$$

else

$$
\left(\Phi_{n}, \Psi_{n}\right) \leftarrow \operatorname{UpdateCurrent}\left(X_{n}, N, \Phi_{n-1}, \Psi_{n-1}\right)
$$

end if

$\hat{\boldsymbol{\theta}} \leftarrow \Phi_{n} \Psi_{n}^{\top} \boldsymbol{Y}_{n}$ 


\subsection{Efficient Online Projection Estimator17}

Whenever new data comes in, the algorithm augments the design matrix by adding one new row to $\Psi_{n-1}$ based on the new observation $X_{n}$. The new row $\left[\psi_{1}\left(X_{n}\right), \psi_{2}\left(X_{n}\right), \ldots, \psi_{N}\left(X_{n}\right)\right]$ can be understood as the embedding of $X_{n}$ into the feature space spanned by $\left(\psi_{j}\right)_{j=1}^{N}$.

When $n=\left\lfloor(N+1)^{\frac{2 \alpha+d}{d}}\right\rfloor$, this algorithm additionally adds a new column to the design matrix $\Psi_{n}$ (increasing the dimension of the basis function we project upon by 1). This new column is just the evaluation of $\psi_{N+1}$ at $\left(X_{i}\right)_{i=1}^{n}$. Recall that $\psi_{N+1}$ is the $(N+1)$-th eigenfunction in Mercer expansion 2.12). It is straightforward to show that this criterion of adding new basis functions ensures $N=\Theta\left(n^{\frac{d}{2 \alpha+d}}\right)$.

The computational expense of each update using Algorithm 1 is

$n^{\frac{2 \alpha+3 d}{2 \alpha+d}}$. In particular, calculating $\Psi_{n}^{\top} \Psi_{n}$ takes $\sim n N^{2} \sim n^{\frac{2 \alpha+3 d}{2 \alpha+d}}$ computation. While this algorithm would give a statistically rate-optimal estimator, and is straightforward to implement, it is rather computationally expensive. In particular, the functional SGD algorithm has a comparatively smaller computational cost of $\sim n$ per update.

\subsection{Efficient Online Projection Estimator}

In this section we explicitly give our proposed method (the details of which are given in Algorithm 2). By using some common block/rank-one updat- 


\subsection{Efficient Online Projection Estimator18}

ing tools from linear algebra, we are able to substantially improve Algorithm 1. In particular, it is expensive to repeatedly calculate $\left(\Psi_{n}^{\top} \Psi_{n}\right)^{-1}$ directly. However, matrix $\Psi_{n}$ has only one more row and (sometimes) one more column than $\Psi_{n-1}$. It is possible to calculate $\left(\Psi_{n}^{\top} \Psi_{n}\right)^{-1}$ by updating $\left(\Psi_{n-1}^{\top} \Psi_{n-1}\right)^{-1}$. The latter will already have been calculated when observing $\left(X_{n-1}, Y_{n-1}\right)$.

When $\Psi_{n}$ has one more row than $\Psi_{n-1}$ :

$$
\Psi_{n}=\left[\begin{array}{c}
\Psi_{n-1} \\
\psi_{n}^{\top}
\end{array}\right]
$$

where $\boldsymbol{\psi}_{n}=\left[\psi_{1}\left(X_{n}\right), \psi_{2}\left(X_{n}\right), \ldots, \psi_{N}\left(X_{n}\right)\right]^{\top}$. We can write $\Psi_{n}^{\top} \Psi_{n}$ in the form:

$$
\Psi_{n}^{\top} \Psi_{n}=\Psi_{n-1}^{\top} \Psi_{n-1}+\boldsymbol{\psi}_{n} \boldsymbol{\psi}_{n}^{\top}
$$

So $\left(\Psi_{n}^{\top} \Psi_{n}\right)^{-1}$ can be calculated from $\left(\Psi_{n-1}^{\top} \Psi_{n-1}\right)^{-1}$ and $\boldsymbol{\psi}_{n}$ by the ShermanMorrison formula (Sherman and Morrison, 1950).

When $\Psi_{n}$ has one more column than $\Psi_{n-1}$ :

$$
\Psi_{n}=\left[\begin{array}{ll}
\Psi_{n-1} & \psi^{N+1}
\end{array}\right]
$$

We can write $\Psi_{n}^{\top} \Psi_{n}$ in the form:

$$
\Psi_{n}^{\top} \Psi_{n}=\left[\begin{array}{cc}
\Psi_{n-1}^{\top} \Psi_{n-1} & \Psi_{n-1}^{\top} \boldsymbol{\psi}^{N+1} \\
\left(\boldsymbol{\psi}^{N+1}\right)^{\top} \Psi_{n-1} & \left(\boldsymbol{\psi}^{N+1}\right)^{\top} \boldsymbol{\psi}^{N+1}
\end{array}\right]
$$


3.4 Computational Expense of Algorithm 219

So $\left(\Psi_{n}^{\top} \Psi_{n}\right)^{-1}$ can be related to $\left(\Psi_{n-1}^{\top} \Psi_{n-1}\right)^{-1}$ by the block matrix inversion formula (Petersen and Petersen, 2008).

The detailed updating rule of the proposed method is given explicitly in Algorithm 2. The basic structure of this algorithm is identical to Algorithm 1, however the updating rules discussed above are used to avoid recalculating some quantities from scratch. We also establish a recursive relationship between $\hat{\boldsymbol{\theta}}_{n+1}$ and $\hat{\boldsymbol{\theta}}_{n}$. Curiously, the recursive formula has a form very similar to pre-conditioned SGD estimator (with the inverse of Gram matrix as the pre-conditioner). When $n \neq\left\lfloor(N+1)^{\frac{2 \alpha+d}{d}}\right\rfloor$, the recursion is:

$$
\hat{\boldsymbol{\theta}}_{n}=\hat{\boldsymbol{\theta}}_{n-1}+\Phi_{n} \boldsymbol{\psi}_{n}\left[Y_{n}-\hat{f}_{n-1, N}\left(X_{n}\right)\right]
$$

Note that for SGD the updating rule replaces $\Phi_{n}$ by $I$ the identity matrix, i.e. omitting the correlation of $\psi_{j}$ 's w.r.t. the empirical measure. When features are added, there is still a geometrical interpretation, we present the result in Appendix S3.

\subsection{Computational Expense of Algorithm 2}

We now show that the computational expense of the updating rule in Algorithm 2 is on average $O\left(n^{\frac{2 d}{2 \alpha+d}}\right)$.

When $n \neq\left\lfloor(N+1)^{\frac{2 \alpha+d}{d}}\right\rfloor$, we do not add a new feature $\psi_{N+1}$ but only update the $\Phi_{n-1}$ matrix with the current $N$ features. The most expensive 
3.4 Computational Expense of Algorithm 220

Algorithm 2 Rule for updating $\hat{\boldsymbol{\theta}}$ with a new observation $\left(X_{n}, Y_{n}\right)$ efficiently. At step *, the value of $\Psi_{n-1}^{\top} \boldsymbol{Y}_{n-1}$ stored in memory needs to be used to avoid repeating calculation.

Input: $\left(X_{i}\right)_{i=1}^{n}, Y_{n}, N, \Phi_{n-1}, \Psi_{n-1}, a, \Psi_{n-1}^{\top} Y_{n-1}$

function UpdateCurrent $\left(X_{n}, N, \Phi_{n-1}, \Psi_{n-1}\right)$ output $\left(\Phi_{n}, \Psi_{n}\right)$

$$
\begin{aligned}
& \boldsymbol{\psi}_{n} \leftarrow\left[\psi_{1}\left(X_{n}\right), \psi_{2}\left(X_{n}\right), \ldots, \psi_{N}\left(X_{n}\right)\right]^{\top} \\
& \Psi_{n} \leftarrow\left[\Psi_{n-1}^{\top} \boldsymbol{\psi}_{n}\right]^{\top}, \Phi_{n} \leftarrow \Phi_{n-1}-\frac{\Phi_{n-1} \psi_{n} \psi_{n}^{T} \Phi_{n-1}}{1+\boldsymbol{\psi}_{\boldsymbol{n}}{ }^{T} \Phi_{n-1} \psi_{\boldsymbol{n}}}
\end{aligned}
$$

function AddBasis $\left(\left(X_{i}\right)_{i=1}^{n}, N, \Phi_{n}, \Psi_{n}\right)$ output $\left(\Phi_{n}, \Psi_{n}\right)$

$$
\begin{aligned}
& \boldsymbol{\psi}^{N+1} \leftarrow\left[\psi_{N+1}\left(X_{1}\right), \psi_{N+1}\left(X_{2}\right), \ldots, \psi_{N+1}\left(X_{n}\right)\right]^{\top} \\
& c \leftarrow\left(\boldsymbol{\psi}^{N+1}\right)^{\top} \boldsymbol{\psi}^{N+1} \quad \boldsymbol{b} \leftarrow \Psi_{n}^{\top} \boldsymbol{\psi}^{N+1} \quad k \leftarrow c-\boldsymbol{b}^{\top} \Phi_{n} \boldsymbol{b} \\
& \Psi_{n} \leftarrow\left[\begin{array}{ll}
\Psi_{n} & \boldsymbol{\psi}^{N+1}
\end{array}\right], \Phi_{n} \leftarrow\left[\begin{array}{cc}
\Phi_{n}+\frac{1}{k} \Phi_{n} \boldsymbol{b} \boldsymbol{b}^{T} \Phi_{n} & -\frac{1}{k} \Phi_{n} \boldsymbol{b} \\
-\frac{1}{k} \boldsymbol{b}^{T} \Phi_{n} & \frac{1}{k}
\end{array}\right] \\
& \left(\Phi_{n}, \Psi_{n}\right) \leftarrow \operatorname{UpdateCurrent}\left(X_{n}, N, \Phi_{n-1}, \Psi_{n-1}\right) \\
& \text { if } n=\text { Floor }\left((N+1)^{2 a+1}\right) \operatorname{then} \\
& \quad\left(\Phi_{n}, \Psi_{n}\right) \leftarrow \text { AddBasis }\left(\left(X_{i}\right)_{i=1}^{n}, N, \Phi_{n}, \Psi_{n}\right) \\
& \quad N \leftarrow N+1
\end{aligned}
$$

end if

$$
\hat{\boldsymbol{\theta}} \leftarrow \Phi_{n} \Psi_{n}^{\top} \boldsymbol{Y}_{n} \quad *
$$

step is the inner product of $\Phi_{n-1}$ and $\boldsymbol{\psi}_{\boldsymbol{n}}$, which is an $N \times N$ matrix multiplied by a $N \times 1$ vector. Since the $N=\Theta\left(n^{\frac{d}{2 \alpha+d}}\right)$ at step $n$, the update is 
3.4 Computational Expense of Algorithm 221

of order $n^{\frac{2 d}{2 \alpha+d}}$.

When $n=\left\lfloor(N+1)^{\frac{2 \alpha+d}{d}}\right\rfloor$, we add both a column and a row to the design matrix $\Psi_{n-1}$. The most expensive step is calculating the vector $\boldsymbol{b}$, which gives the pair-wise inner product between $\psi_{N+1}$ and $\left(\psi_{j}\right)_{j=1}^{N}$ with respect to empirical measure. In this step an $N \times(n-1)$ matrix is multiplied by an $(n-1) \times 1$ vector, which requires computation of order $n^{\frac{2 \alpha+2 d}{2 \alpha+d}}$. However the algorithm adds new features less frequently as $n$ increases. Thus, in calculating average computational cost, we amortize this expense over all the updates between the inclusion of new basis functions.

Let

$$
\begin{gathered}
n=(N)^{\frac{2 \alpha+d}{d}} \\
n^{+}=(N+1)^{\frac{2 \alpha+d}{d}}
\end{gathered}
$$

that is, $n$ is the first step when there are more than $N$ features included; $n^{+}$is the first step when there are more than $N+1$ features. Then the length of the interval between the two "basis adding" steps is

$$
\begin{aligned}
n^{+}-n & =(N+1)^{\frac{2 \alpha+d}{d}}-(N)^{\frac{2 \alpha+d}{d}} \\
& =\Theta\left(N^{2 \alpha / d}\right)=\Theta\left(n^{\frac{2 \alpha}{2 \alpha+d}}\right)
\end{aligned}
$$

Thus, $O\left(n^{\frac{2 \alpha+2 d}{2 \alpha+d}}\right)$ computation is done per $n^{\frac{2 \alpha}{2 \alpha+d}}$ steps, which is, on average, $O\left(n^{\frac{2 d}{2 \alpha+d}}\right)$ per step. Thus the average computational expense of a single update with Algorithm 2 is of order $n^{\frac{2 d}{2 \alpha+d}}$. 


\section{Theoretical Analysis of the Online Projection Estimator}

In this section, we formally show that the online estimator in this paper achieves the optimal statistical convergence rate when the true regression function belongs to the hypothesized RKHS. In previous theoretical analysis of (batch) projection estimators (Tsybakov, 2008), the proof is shown when $\psi_{j}$ 's are orthogonal to each other w.r.t. the empirical measure of the covariates. This event has probability zero if $X$ has a continuous density. In this section, we show it is possible to get a rate-optimal bound on the generalization error of $\hat{f}_{n, N}$ even if $\psi_{j}$ 's (the eigenfunctions of the kernel with respect to our "convenient" working distribution) are quite correlated w.r.t. the empirical measure of $X$.

Recall that $\mathcal{F}_{N}=\operatorname{span}\left(\psi_{1}, \ldots, \psi_{N}\right)$ is the linear space spanned by the first $N$ eigenfunctions. Define the population minimizer $f_{N}$ over $\mathcal{F}_{N}$ as

$$
f_{N}:=\arg \min _{f \in \mathcal{F}_{N}} \mathbb{E}\left[\left(f(X)-f_{\rho}(X)\right)^{2}\right]
$$

Here we remind our reader that $\hat{f}_{n, N} \in \mathcal{F}_{N}$ is the estimator, $f_{N}$ is the population risk minimizer over $\mathcal{F}_{N}$ and $f_{\rho} \in \mathcal{H}$ is the target function to be estimated. To establish the result that $\left\|\hat{f}_{n, N}-f_{\rho}\right\|_{2} \rightarrow 0$ as $n \rightarrow \infty$, we first bound the rate at which $\left\|\hat{f}_{n, N}-f_{N}\right\|_{2}$ goes to 0 as $N$ grows (sufficiently slowly); then we bound the rate at which $\left\|f_{N}-f_{\rho}\right\|_{2} \rightarrow 0$ as $N \rightarrow \infty$. With 
the correct choice of $N=\Theta\left(n^{\frac{d}{2 \alpha+d}}\right)$ we can balance the rate of the above two term converging to 0 . Before we state the result, we give assumptions necessary for the proof.

(A1) The joint distribution of i.i.d. $\left(X_{i}, Y_{i}\right)$ has support $\mathcal{X} \times \mathbb{R} \subset$ $\mathbb{R}^{d} \times \mathbb{R}$ and $\mathcal{X}$ is compact. The i.i.d. zero-mean noise random variables $\epsilon_{i}=Y_{i}-f_{\rho}\left(X_{i}\right)$ satisfy the following

$$
\left\|\epsilon_{i}\right\|_{m, 1}:=\int_{0}^{\infty} \mathbb{P}\left(\left|\epsilon_{i}\right|>t\right)^{1 / m} d t<\infty \quad \text { for some } m>1
$$

Note. If for some $\delta>0, m>1$ we have that $m+\delta$ moment of $\epsilon_{i}$ exists, then (A1) is satisfied for that value of $m$. This is slightly stronger than existence of the $m$-th moment, cf. (Ledoux and Talagrand, 2013, Chapter 10)

Our noise assumption is substantially weaker than the typical subGaussian noise assumptions (sub-Gaussian random variables have all moments bounded). In the light tail noise setting, the level of the noise only influences the convergence speed by at most a constant. However we will see in Theorem 3, if the eigenvalues decrease too fast (the RKHS is too small) and the noise has too few moments, the convergence rate will depend on the noise level. Our analysis characterizes the interplay between the size of RKHS space and the noise level, using a sharp multiplier inequality (Han et al., 2019, Theorem 1). There are currently no other methodologies, to our knowledge, that are both computationally tractable and have provable con- 
vergence guarantees with heavy-tailed noise in the online non-parametric regression setting.

(A2) The true regression function $f_{\rho}$ belongs to the known RKHS $\mathcal{H}$ i.e. the RKHS-norm $\left\|f_{\rho}\right\|_{\mathcal{H}}$ is finite.

(A3) The kernel function has Mercer expansion $K(x, z)=\sum_{j=1}^{\infty} \lambda_{j} \psi_{j}(x) \psi_{j}(z)$, where $\left(\psi_{j}\right)_{j=1}^{\infty}$ are orthonormal with respect to some specified working distribution $\bar{\rho}_{X}$, and $\lambda_{j}=\Theta\left(j^{-2 \alpha / d}\right)$ with $\alpha>d / 2$.

(A4) The distribution of $X, \rho_{X}$, is absolutely continuous w.r.t. $\bar{\rho}_{X}$. Let $p_{X}=d \rho_{X} / d \bar{\rho}_{X}$ denote its Radon-Nikodym derivative. We assume for some $D<\infty:$

$$
p_{X}(x) \leq D \text { for all } x \in \mathcal{X}
$$

Note. In the (very common) case that both of these have densities with respect to Lebesgue measure, this is equivalent the ratio of their densities being bounded.

Theorem 3 (Optimal convergence rate). Assume (A1-A4), let $\hat{f}_{n, N}$ be the projection estimator (3.17). Assume that $\left\|\hat{f}_{n, N}\right\|_{\infty} \leq M$, for some $M<\infty$. Choosing $N=\Theta\left(n^{\frac{d}{2 \alpha+d}}\right)$, we have

$$
\left\|\hat{f}_{n, N}-f_{\rho}\right\|_{2}=O_{P}\left(n^{-\frac{\alpha}{2 \alpha+d}} \sqrt{\log n} \vee n^{-\frac{1}{2}+\frac{1}{2 m}} \sqrt{\log n}\right)
$$


If $m \geq 2$ in (A1), the above bound holds in expectation:

$$
\mathbb{E}\left[\left\|\hat{f}_{n, N}-f_{\rho}\right\|_{2}\right]=O\left(n^{-\frac{\alpha}{2 \alpha+d}} \sqrt{\log n} \vee n^{-\frac{1}{2}+\frac{1}{2 m}} \sqrt{\log n}\right)
$$

Note as long as all the moments of $\epsilon_{i}$ 's exist (e.g. when $\epsilon_{i}$ 's are subexponential), the convergence rate only depends on the size of the RKHS. One merit of our method is that even if the noise does not have finite variance, that is, $m<2$ in (A1), our method still has convergence guarantees. To our knowledge, existing work on non-parametric SGD does not give convergence guarantees with such heavy tailed noise.

As we compare the two components on the RHS of the bound presented in 4.26 , we can see that when $m>\frac{2 \alpha}{d}+1$, that is, when we have a relative light-tailed noise, our bound is dominated by the size of the RKHS. However when $m<\frac{2 \alpha}{d}+1$, it is the noise that dominates our bound. Also note that as $d$ increases, fewer moments on $\epsilon$ are required for our bound to match the classical non-parametric minimax rate in our RKHS.

The following lower bound demonstrates that this rate of convergence is indeed optimal (up to a logarithm term) among all estimators. For $\lambda_{j}=$ $\Theta\left(j^{-2 \zeta}\right)$ (to compare with Theorem 3, take $\left.\zeta=\alpha / d\right)$, let $B_{R}=\{f \in$ $\left.\mathcal{H} \mid\|f\|_{\mathcal{H}} \leq R\right\}$ be the $R$-ball in RKHS $\mathcal{H}$. Then we have the minimax 
bound:

$$
\liminf _{n \rightarrow \infty} \inf _{\hat{f}} \sup _{f_{\rho} \in B_{R}} \mathbb{E}\left[n^{\frac{\zeta}{2 \zeta+1}}\left\|\hat{f}-f_{\rho}\right\|_{2}\right] \geq C
$$

where the infimum ranges over all possible functions $\hat{f}$ that are measurable of the data. For a derivation of the lower bound, see Wainwright (2019, Chap. 15).

Upper bounds similar to our results in Theorem 3 have been shown in Tarres and Yao (2014); Dieuleveut and Bach (2016), for SGD-type nonparametric online methods. However the proposed estimators there use $n$ basis function, therefore have an unacceptable $\Theta\left(n^{2}\right)$ total computational expense. There are methods that aim to improve the computational aspect by using random features or other acceleration methods (cf. Section 2.1), however the theoretical guarantees on statistical convergence rates in that work are generally quite weak (generally giving upper bounds of $n^{-1 / 4}$ in RMSE, which is far from the minimax rate) and insensitive to the decay rate of eigenvalues.

Many existing online nonparametric estimators aim to find a function $f \in \mathcal{F}$ that minimizes an expected convex loss $\mathbb{E}[l(f(X), Y)]$, which is a more general setting than this study. However, the majority of previous work on this topic assumes the loss function $l(\cdot, \cdot)$ is Lipschitz w.r.t. the first argument, including Dai et al. (2014); Si et al. (2018); Koppel et al. (2019); 
Lu et al. (2016). Specializing to the regression problem (with squared-errorloss), this is essentially assuming the outcomes $Y_{i}$ (therefore the noise $\epsilon_{i}$ ) are uniformly bounded: because $l(f(x), y)-l(f(z), y)=(f(x)-y)^{2}-(f(z)-$ $y)^{2}=(f(x)-f(z))(f(x)+f(z)-2 y)$. If we require $l(\cdot, \cdot)$ to be Lipschitz, we basically require $f(x), f(z), y$ to be uniformly bounded. Although we still only consider bounded $f$ in this work, we relax the contraint on the noise variables: we require only finite moments of $\epsilon_{i}$ and show (in)sensitivity of our bound.

\section{Multivariate Regression Problems}

In most applications, the covariate $X_{i}$ 's take value in $\mathbb{R}^{d}$ where $d>1$. If the kernel function $K: \mathbb{R}^{d} \times \mathbb{R}^{d} \rightarrow \mathbb{R}$ has a known Mercer expansion (2.12), then the proposed method can be applied directly. If the kernel function takes a tensor product form (e.g. the Gaussian kernel), or is constructed from a 1dimension kernel via a tensor product (e.g. $K(x, z)=\prod_{k=1}^{d} \min \left\{x^{(k)}, z^{(k)}\right\}$,

where $x^{(k)}$ is the $k$-th entry of $x \in \mathbb{R}^{d}$ ), the eigenvalues and eigenfunctions are just the tensor product of the 1-dimensional kernels' Michel, 2012, Section 3.5), (Xiu, 2010, Section 5.2). However, as presented in Section 4. the minimax rate of estimating in a $d$-dimension $\alpha$-order Sobolev space is $\Theta\left(n^{-\frac{\alpha}{2 \alpha+d}}\right)$, which becomes quite slow when $d$ is large (unless at the same 
time a large $\alpha$ is assumed).

A popular low-dimension structure people have used is the nonparametric additive model (Hastie et al., 2009; Yuan and Zhou, 2016), which is thought to effectively balance model flexibility and interpretability. For $x \in$ $\mathbb{R}^{d}$, we might consider imposing an additive structure on our model (1.1):

$$
f_{\rho}(x)=\sum_{k=1}^{d} f_{\rho, k}\left(x^{(k)}\right)
$$

where the component functions $f_{\rho, k}$ belong to a RKHS $\mathcal{H}$ (in general they can belong to different spaces). For a fixed $d$, the minimax rate for estimating an additive model is identical (up to a multiplicative constant $d$ ) to the minimax rate in the analogous one-dimension nonparametric regression problem that works with the same hypothesis space $\mathcal{H}$ Raskutti et al. 2009). The proposed online method can be directly generalized to this setting, for more discussion and empirical performance, see Appendix S4.

\section{Simulation Study}

In this section, we illustrate both the computational and statistical efficiency of the online projection estimator, in both one-dimension regression and additive model settings. 
6.1 Generalization Error of the Online Projection Estimator is Rate-Optimal29

\subsection{Generalization Error of the Online Projection Estimator is Rate-Optimal}

In this section, we use simulated data to illustrate that the generalization error of our estimator reaches the minimix-optimal rate. For each sample, $X_{i}$ is generated from $\rho_{X}$ whose density function is $p_{X}(x) ; Y_{i}$ is generated by $Y_{i}=f_{\rho}\left(X_{i}\right)+\epsilon_{i}$. The details of the parameters are listed in Table 1. In example 1 , we purposely select $\rho_{X}$ such that $\int_{0}^{1} \psi_{i}(x) \psi_{j}(x) p_{X}(x) d x=\delta_{i j}$, together with bounded noise. In example 2, basis functions are no longer orthogonal w.r.t. $\rho_{X}$ and a low signal-noise ratio is applied. In both simple and more realistic scenarios, the online projection estimator achieves rateoptimal statistical convergence.

The $f_{\rho}$ in example 1 is taken from Dieuleveut and Bach (2016), where they used it to illustrate the performance of the functional SGD estimator; the regression function in example 2 is also used in a study of wavelet neural networks (Alexandridis and Zapranis, 2013).

In example 1, the hypothesis space is the second-order spline on the circle

$$
\begin{aligned}
& W_{2}^{0}(\text { per })=\left\{f \in L^{2}([0,1]) \mid \int_{0}^{1} f(u) d u=0\right. \\
& \left.\quad f(0)=f(1), f^{\prime}(0)=f^{\prime}(1), \int_{0}^{1}\left(f^{\prime \prime}(u)\right)^{2} d u<\infty\right\}
\end{aligned}
$$

In example 2, we use Sobolev space $W_{1}^{0}([0,1])$ defined in 2.10). Because 


\subsection{Generalization Error of the Online Projection Estimator is}

Rate-Optimal30

Table 1: Settings of simulation studies. ${ }^{*} B_{4}(x)=x^{4}-2 x^{3}+x^{2}-\frac{1}{30}$ is the 4-th Bernoulli polynomial, and $\{x\}$ means taking the fractional part of $x$.

\begin{tabular}{|ccc|}
\hline & Example 1 & Example 2 \\
\hline Kernel $K(s, t)$ & $\frac{-1}{24} B_{4}(\{s-t\})^{*}$ & $\min \{s, t\}$ \\
Eigenvalue $\lambda_{j}$ & $\frac{2}{(2 \pi j)^{4}}=O\left(j^{-4}\right)$ & $\frac{4}{(2 j-1)^{2} \pi^{2}}=O\left(j^{-2}\right)$ \\
Basis function $\psi_{j}(x)$ & $\sin (2 \pi j x), \cos (2 \pi j x)$ & $\sqrt{2} \sin \left(\frac{(2 j-1) \pi x}{2}\right)$ \\
$p_{X}(x)$ & $1_{[0,1]}(x)$ & $(x+0.5) 1_{[0,1]}(x)$ \\
Noise $\epsilon$ & $\mathrm{Unif}([-0.02,0.02])$ & $\operatorname{Normal}(0,5)$ \\
& & \\
True regression function $f_{\rho}$ & $B_{4}(x)$ & \\
& $+\cos ^{2}(12 x-6)$ & \\
\hline
\end{tabular}

eigenvalues decrease faster in example 1, we observe a convergence rate of $\sim n^{-4 / 5}$, which is faster that that in example $2, \sim n^{-2 / 3}$.

We use $\left\|\hat{f}_{n, N}-f_{\rho}\right\|_{2}^{2}$ as a measure of goodness of fitting (Figure 1 ). The method in this paper is compared with an online nonparametric SGD estimator (Dieuleveut and Bach, 2016) and the kernel ridge regression (KRR) estimator (1.4). Although KRR might have a better generalization capacity (the rates should be the same, but there might be an improvement in the constant), it is computationally prohibitive to apply it in the online learning 


\subsection{CPU Time31}

- SGD - This paper - KRR

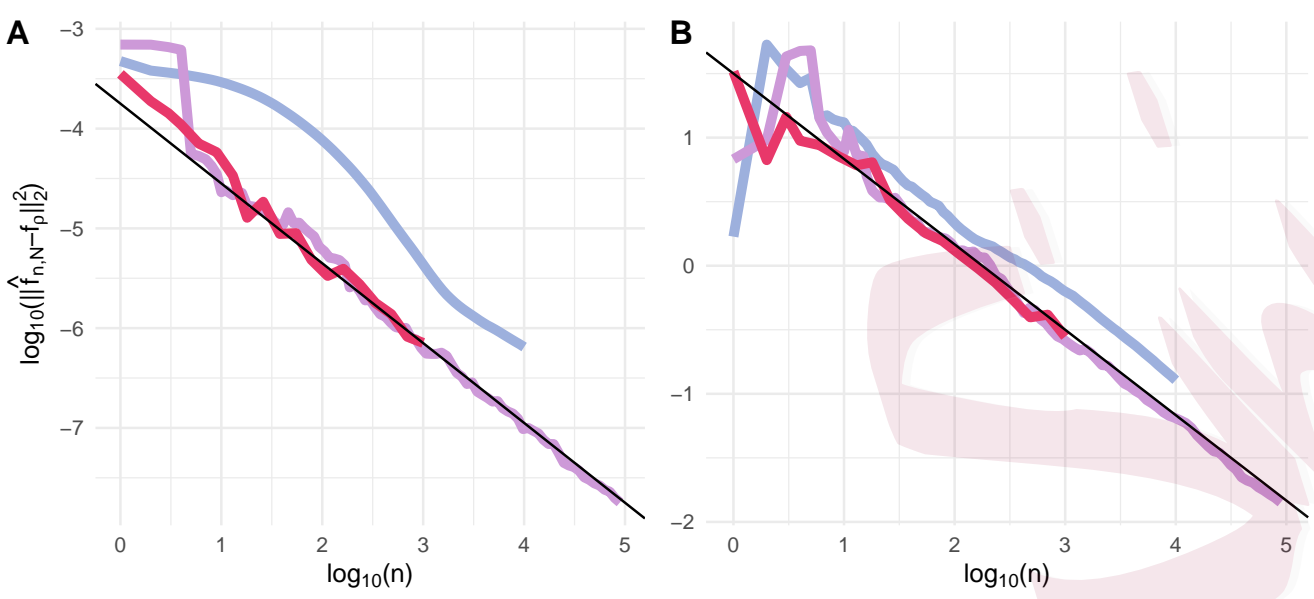

Figure 1: $\log _{10}\left\|\hat{f}_{n, N}-f_{\rho}\right\|_{2}^{2}$ against $\log _{10} n .(\mathbf{A})$ Example 1, black line has slope $=-4 / 5 ;$ (B) Example 2, black line has slope $=-2 / 3$. Each curve is calculated as the average of 15 repetitions. Due to different computational costs, we chose different maximum $n$ for different methods.

setting, so we only include this method as a reference. The hyperparameters for each method are chosen to optimize performance (oracle hyperparameters). For our method, it is the constant in front of timing for adding new basis functions. In Figure 2, we present several typical realizations of $\hat{f}_{n, N}$ for both examples, together with data points. 


\subsection{CPU Time32}

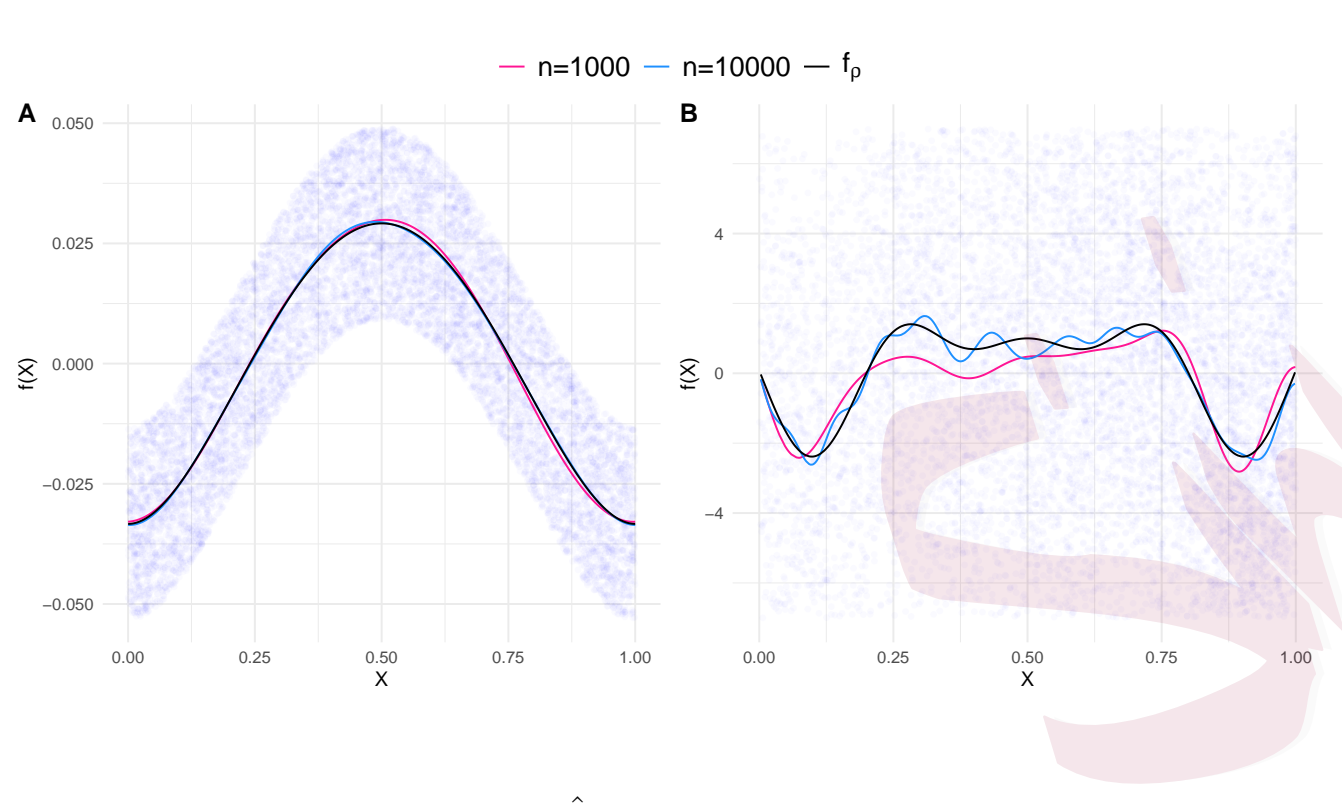

Figure 2: Realizations of $\hat{f}_{n, N}$. (A) Example 1; (B) Example 2.

\subsection{CPU Time}

Figure 3 shows the CPU time used in calculating online estimators for up to $n$ samples when solving example 2 , for the online projection estimator and nonparametric SGD estimator. Experiments were run on a computer with 1 Intel Core m3 processor, $1.2 \mathrm{GHz}$, with $8 \mathrm{~GB}$ of RAM. For the projection estimator, new basis functions are added when $n=\left\lfloor N^{2 \alpha+1}\right\rfloor, N=1,2, \ldots$. First, we can see, for all $\alpha \in\{1,2,3\}$, the online projecting estimators are all significantly faster to compute than nonparametric SGD estimator after $n>10^{4}$, because the latter requires evaluation of $n$ basis functions for the $n+1$ st update, which will accumulate very fast. In addition, for larger $\alpha$ the 


\subsection{CPU Time33}

total computational cost for the online projection estimator becomes nearly linear in $n$. There are also some "jumps" in the CPU time for the online projection estimator: They correspond to steps when new basis functions are added in. Both of the phenomena match our analysis in Section 3.4 . Although it seems beneficial both computationally and statistically to use a larger $\alpha$, it is important to remember that $\alpha$ too large may result in poor generalization error - this occurs if the RKHS associated with $\alpha$ becomes so small that it no longer includes $f_{\rho}$ (see discussion in Simon and Shojaie (2018)).

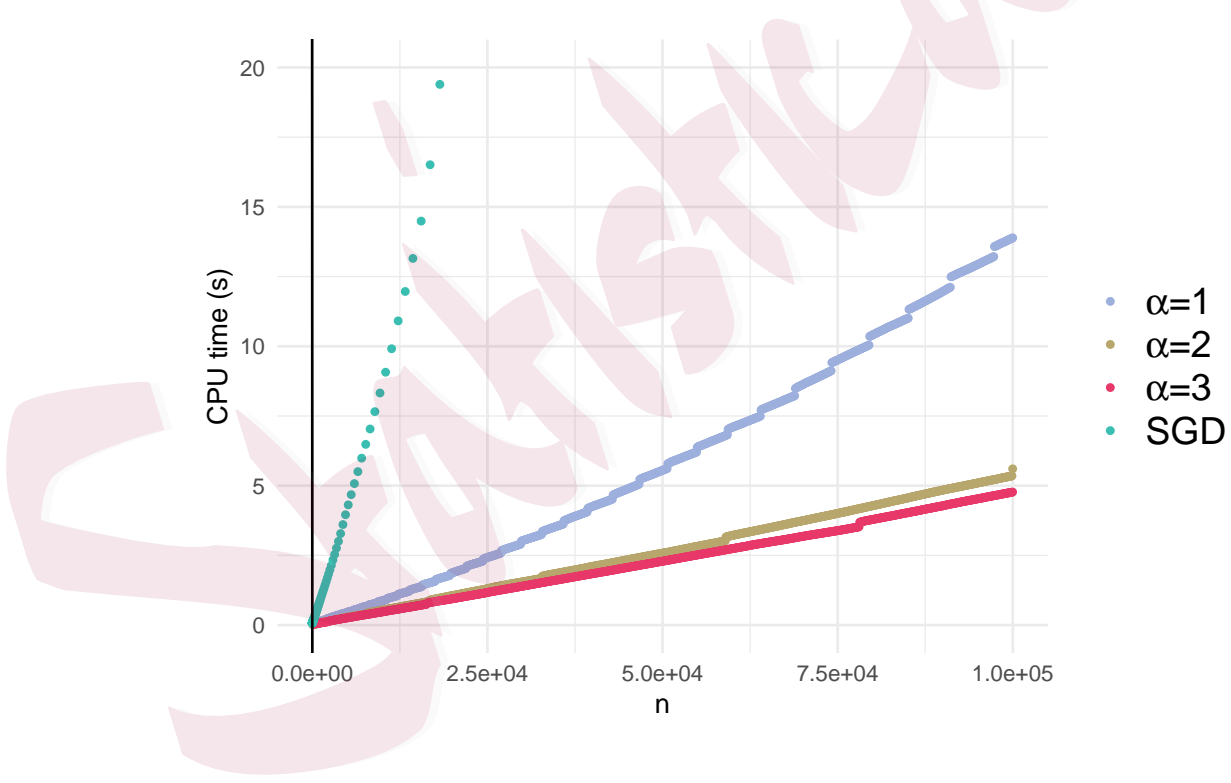

Figure 3: CPU time against sample size (10 runs each curve). 


\section{Discussion}

In this paper, we proposed a framework to construct online nonparametric regression estimators when the hypothesis space is a RKHS. We showed that: (i) the error of the proposed estimator is near-optimal; and (ii) the computational expense of calculating such estimators is much lower than other contemporary estimators with similar statistical guarantees. In addition, our estimator is actually precisely an empirical risk minimizer (in a linear space of slowly growing dimension), which allows us to give theoretical guarantees when the noise is heavy tailed (as compared to the previously required assumptions of boundedness).

In this work, we leveraged properties of least-squares loss to efficiently update the empirical risk minimizer $\hat{f}_{n, N}$ in an online manner. However, for a general convex loss function (e.g. logistic regression), construction of an online nonparametric estimator that has both guaranteed optimal generalization capacity and is computationally feasible for larger problems is still an open question. Although there are functional SGD type estimators designed for this purpose (discussion in Section 2.1), it would be interesting to see if it is possible to design estimators that are both computationally efficient to update and are (approximate) ERM in a deterministic space. 


\section{Supplementary Materials}

In the Appendix, we provide provide proof of Theorem 3 . In the later sections, we give a complete description of settings for simulations from the main text, together with more examples. We also include some additional discussion on the applications of our estimator.

\section{Acknowledgements}

N.S and T.Z. were both supported by NIH grant R01HL137808.

\section{References}

Alexandridis, A. K. and Zapranis, A. D. (2013). Wavelet neural networks: A practical guide. Neural Networks, 42:1-27.

Babichev, D. and Bach, F. (2018). Constant step size stochastic gradient descent for probabilistic modeling. stat, 1050:21.

Bach, F. and Moulines, E. (2013). Non-strongly-convex smooth stochastic approximation with convergence rate o $(1 / \mathrm{n})$. In Advances in neural information processing systems, pages 773-781.

Belloni, A., Chernozhukov, V., and Wang, L. (2014). Pivotal estimation via square-root lasso in nonparametric regression. The Annals of Statistics, $42(2): 757-788$. 


\section{REFERENCES36}

Christmann, A. and Steinwart, I. (2008). Support vector machines.

Cucker, F. and Smale, S. (2002). On the mathematical foundations of learning. Bulletin of the American mathematical society, 39(1):1-49.

Dai, B., Xie, B., He, N., Liang, Y., Raj, A., Balcan, M.-F. F., and Song, L. (2014). Scalable kernel methods via doubly stochastic gradients. In Advances in Neural Information Processing Systems, pages 3041-3049.

Dieuleveut, A. and Bach, F. (2016). Nonparametric stochastic approximation with large step-sizes. The Annals of Statistics, 44(4):1363-1399.

Fasshauer, G. E. and McCourt, M. J. (2015). Kernel-based approximation methods using Matlab, volume 19. World Scientific Publishing Company.

Frostig, R., Ge, R., Kakade, S. M., and Sidford, A. (2015). Competing with the empirical risk minimizer in a single pass. In Conference on learning theory, pages 728-763.

Gittens, A. and Mahoney, M. W. (2016). Revisiting the nyström method for improved large-scale machine learning. The Journal of Machine Learning Research, 17(1):3977-4041.

Han, Q., Wellner, J. A., et al. (2019). Convergence rates of least squares 
regression estimators with heavy-tailed errors. Annals of Statistics, $47(4): 2286-2319$.

Härdle, W., Kerkyacharian, G., Picard, D., and Tsybakov, A. (2012). Wavelets, approximation, and statistical applications, volume 129. Springer Science \& Business Media.

Hastie, T., Tibshirani, R., and Friedman, J. (2009). The elements of statistical learning: data mining, inference, and prediction. Springer Science \& Business Media.

Koppel, A., Warnell, G., Stump, E., and Ribeiro, A. (2019). Parsimonious online learning with kernels via sparse projections in function space. The Journal of Machine Learning Research, 20(1):83-126.

Kushner, H. and Yin, G. G. (2003). Stochastic approximation and recursive algorithms and applications, volume 35. Springer Science \& Business Media.

Ledoux, M. and Talagrand, M. (2013). Probability in Banach Spaces: isoperimetry and processes. Springer Science \& Business Media.

Leoni, G. (2017). A first course in Sobolev spaces. American Mathematical Soc. 


\section{REFERENCES38}

Liu, F., Huang, X., Chen, Y., and Suykens, J. A. (2020). Random features for kernel approximation: A survey in algorithms, theory, and beyond. arXiv preprint arXiv:2004.11154.

Lu, J., Hoi, S. C., Wang, J., Zhao, P., and Liu, Z.-Y. (2016). Large scale online kernel learning. The Journal of Machine Learning Research, 17(1):1613-1655.

Michel, V. (2012). Lectures on Constructive Approximation: Fourier, Spline, and Wavelet Methods on the Real Line, the Sphere, and the Ball. Springer Science \& Business Media.

Petersen, K. B. and Petersen, M. S. (2008). The matrix cookbook. Technical University of Denmark, 7(15):510.

Raskutti, G., Yu, B., and Wainwright, M. J. (2009). Lower bounds on minimax rates for nonparametric regression with additive sparsity and smoothness. In Advances in Neural Information Processing Systems, pages $1563-1570$.

Rudi, A. and Rosasco, L. (2017). Generalization properties of learning with random features. In Advances in Neural Information Processing Systems, pages $3215-3225$. 
Santin, G. and Schaback, R. (2016). Approximation of eigenfunctions in kernel-based spaces. Advances in Computational Mathematics, 42(4):973993.

Schölkopf, B., Herbrich, R., and Smola, A. J. (2001). A generalized representer theorem. In International conference on computational learning theory, pages 416-426. Springer.

Sherman, J. and Morrison, W. J. (1950). Adjustment of an inverse matrix corresponding to a change in one element of a given matrix. The Annals of Mathematical Statistics, 21(1):124-127.

Si, S., Kumar, S., and Li, Y. (2018). Nonlinear online learning with adaptive nystr $\backslash "\{0\}$ m approximation. arXiv preprint arXiv:1802.07887.

Simon, N. and Shojaie, A. (2018). Convergence rates of nonparametric penalized regression under misspecified smoothness. Statistica Sinica Preprint, No: SS-2018-0144.

Tarres, P. and Yao, Y. (2014). Online learning as stochastic approximation of regularization paths: Optimality and almost-sure convergence. IEEE Transactions on Information Theory, 60(9):5716-5735. 
Tsybakov, A. (2008). Introduction to Nonparametric Estimation. Springer Science \& Business Media.

Wahba, G. (1990). Spline models for observational data, volume 59. Siam.

Wainwright, M. J. (2019). High-dimensional statistics: A non-asymptotic viewpoint, volume 48. Cambridge University Press.

Xiu, D. (2010). Numerical methods for stochastic computations: a spectral method approach. Princeton university press.

Yuan, M. and Zhou, D.-X. (2016). Minimax optimal rates of estimation in high dimensional additive models. The Annals of Statistics, 44(6):25642593.

Department of Biostatistics, University of Washington, Seattle, WA 98195, USA

E-mail: zty@uw.edu

Department of Biostatistics, University of Washington, Seattle, WA 98195, USA

E-mail: nrsimon@uw.edu 